Théologiques

Théologiques

\title{
La tradition mystique chrétienne et la dignité de la personne humaine
}

\section{Rob Faesen}

Volume 18, numéro 2, 2010

Les lieux de la spiritualité aujourd'hui

URI : https://id.erudit.org/iderudit/1007478ar

DOI : https://doi.org/10.7202/1007478ar

Aller au sommaire du numéro

\section{Éditeur(s)}

Faculté de théologie et de sciences des religions, Université de Montréal

\section{ISSN}

1188-7109 (imprimé)

1492-1413 (numérique)

Découvrir la revue

\section{Citer cet article}

Faesen, R. (2010). La tradition mystique chrétienne et la dignité de la personne humaine. Théologiques, 18(2), 15-42. https://doi.org/10.7202/1007478ar
Résumé de l'article

Cet article soutient que la tradition mystique chrétienne peut contribuer de façon importante à la promotion de la dignité de la personne humaine. Puisqu'elle témoigne d'une conscience expérientielle des fondements transcendants du « je » (c'est-à-dire la rencontre de Dieu dans l'amour), l'anthropologie de la tradition contemplative et mystique préfère concevoir le fondement de la personne humaine - l'être de la personne humaine - en tant que relation d'amour transcendant plutôt qu'en termes d'individualité du « je ». Cette relation est unique en soi ; par conséquent, l'anthropologie mystique accorde de la valeur à l'unicité de chaque personne et s'oppose à l'idée qu'un humain puisse n'être qu'une individualité, une composante remplaçable de la collectivité. 


\title{
La tradition mystique chrétienne et la dignité de la personne humaine
}

\author{
Rob FAesen, S.J." \\ Théologie \\ Katholieke Universiteit, Leuven (Belgique)
}

La question soulevée dans cet essai est la suivante ${ }^{1}$ : qu'est-ce que la tradition mystique chrétienne peut nous apprendre sur le cœur de l'humanisme? Il serait tentant de penser que la tradition mystique et l'humanisme sont deux entités très éloignées, voire opposées l'une à l'autre; j'ai pour objectif de démontrer que ceci n'est pas le cas. Je vais procéder en trois étapes: premièrement, je vais définir comment j'entends l'adjectif «mystique » et donc comment l'on peut discerner la spécificité de la tradition mystique chrétienne; deuxièmement, je vais examiner certains des éléments saillants de la compréhension de la personne humaine chez les mystiques chrétiens et, indirectement, de la culture humaine; troisièmement, je vais discuter brièvement de ce que ceci veut dire pour nous aujourd'hui, de la façon dont cette vision nous remet en question dans notre $\mathrm{XxI}^{\mathrm{e}}$ siècle mondialisé et multiculturel. Dans chacune de ces étapes, je m'inspirerai principalement d'Albert Deblaere, S.J. (1916-1994).

* Rob Faesen, S.J. est professeur à la Faculté de théologie de la Katholieke Universiteit Leuven (Belgique) et à la Ruusbroecgenootschap, Faculté des arts, Universiteit Antwerpen (Belgique). Son principal champ de recherche est la littérature mystique médiévale néerlandaise. Il a récemment publié R. Faesen, R. van Nieuwenhove et H. Rolfson (2008), dir., Late Medieval Mysticism of the Low Countries, New York, Paulist Press (Classics of Western Spirituality).

1. Texte traduit de l'anglais par la rédaction. Ceci est une version légèrement révisée d'une conférence donnée à l'Université Sogang (Séoul, Corée du Sud) à l'occasion d'un congrès interreligieux sur les aspects du mysticisme dans des traditions religieuses différentes. Dans la conférence, des affirmations sont faites par rapport au mysticisme chrétien qui seraient évidemment trop simplificatrices pour un public de spécialistes en histoire du mysticisme chrétien; ces affirmations devraient être entendues dans le contexte de cet échange interreligieux.

(C) Revue Théologiques 2010. Tout droit réservé. 


\section{1. «Mystique» et «tradition mystique chrétienne»}

Le mot «mystique» est utilisé très souvent avec plusieurs connotations différentes; pour éviter tout malentendu il est nécessaire que j'en formule ma compréhension. Une des définitions les plus brèves et les plus concises du mot «mystique» est donnée par Albert Deblaere, c'est-à-dire «qui renvoie à une expérience immédiate et passive de la présence de Dieu » (Deblaere 2004, 113). Le terme "mystique» comprend un aspect expérientiel (et donc personnel, puisque toute expérience est personnelle), c'est-à-dire de la présence de Dieu, du Transcendant. On peut penser ou étudier à propos de Dieu mais faire l'expérience de sa présence est quelque chose de très différent. Deblaere précise que pour les mystiques cette expérience de la présence est immédiate et passive: «immédiate " parce qu'il n'y a pas de médiation entre Dieu et la personne humaine (pas de symbole, de mot, d'image ou de concept); "passive » parce qu'elle est donnée à la personne humaine, non pas acquise par elle.

Examinons un exemple de telles expériences dans la vie du célèbre mathématicien Blaise Pascal (1623-1662). Dans la nuit de lundi, le 23 novembre 1654, il eut une expérience qui changea complètement sa vie intérieure. Après cette expérience, il écrivit ces mots sur un morceau de papier qu'il porta sur lui le reste de sa vie. En voici quelques lignes:

L'an de grace 1654.

Lundy $23^{e}$ Nov. [...].

Depuis environ dix heures et demi du soir jusques environ minuit et demi

FEU

Dieu d'Abraham. Dieu d'Isaac. Dieu de Jacob non des philosophes et des scavans.

Certitude joye certitude sentiment joye.

Dieu de Jesus Christ

Deum meum et Deum vestrum Jeh. 20. 17

Ton Dieu sera mon Dieu. Ruth [...]

Grandeur de l'ame humaine $[\ldots]^{2}$.

Il s'agit d'une description d'une expérience soudaine: Blaise Pascal ne s'attendait à rien du tout et il n'y était pas réellement préparé. De plus, il décrit cette expérience comme un réel contact, une rencontre de Dieu dans l'amour.

2. Fac-similé dans Bremond (1938, 368). 
Avant d'aller plus loin, il est important de clarifier ce qui est entendu par «immédiat». Il ne faudrait pas penser que l'expérience immédiate de Dieu fut considérée par les mystiques chrétiens comme «meilleure» que l'expérience vécue à travers la médiation, ou alors que ces mystiques croyaient que l'expérience immédiate discréditait toute médiation. Le mystique Ruusbroec (1293-1381) présentait d'ailleurs une formule équilibrée à cet égard:

Comprenons bien, en effet, Dieu vient sans cesse en nous, par intermédiaire et sans intermédiaire, et qu'il exige que nous jouissions et opérions, sans que l'un n'empêche l'autre, mais plutôt afin que sans cesse l'un fortifie l'autre.

(Opera omnia 3, 533, trad. par Wisques, 192)

L'objet est alors manifeste dans l'immédiat et la médiation; pas dans «un ou l'autre» mais bien dans «l'un et l'autre». Cette observation est importante: il serait faux de penser que les mystiques chrétiens rejettent l'expérience de la médiation. Si c'était le cas, ils suggèreraient alors que le but de la vie soit une telle expérience immédiate de Dieu. Autant que je la comprenne, il me semble que leur conviction indique plutôt que le but de la vie consiste en l'amour (la satisfaction du premier et second commandement) et non pas en une expérience comme telle.

\subsection{Culture contemplative-mystique}

D'innombrables témoignages de soudaines expériences de la présence du Divin sont rapportés dès les débuts de l'histoire de la culture chrétienne. J'ai même tendance à penser que la culture chrétienne du premier millénaire fut profondément marquée par cette dimension. Pour illustrer mon propos, je cite Bède le Vénérable (672/673-735). Dans son commentaire sur la description vétérotestamentaire de la construction du temple de Salomon, il s'exprime ainsi par rapport au verset «Il fit au Temple des fenêtres obliques» (voir 1 R 7,4):

«Il fit au temple des fenêtres obliques ». Les fenêtres du temple sont les saints docteurs et toutes les personnes spirituelles de l'Église, auxquelles il est donné, quand elles se quittent en esprit pour Dieu, de voir plus spécialement que d'autres les arcanes des mystères du ciel. Lorsqu'elles dévoilent publiquement aux fidèles ce qu'elles voient dans le secret, on dit d'elles, qu'elles ont été, pour recevoir la lumière du soleil, une sorte de fenêtres obliques, c'est-à-dire plus larges à l'intérieur; car, il est nécessaire de toute évidence que quiconque perçoit, ne fût-ce qu'un moment, la lumière des réalités supérieures qu'il contemple, dilate aussitôt la capacité de son cœur 
en se mortifiant un peu plus, et, par de diligents exercices, le prépare à en saisir de plus grandes encore. (Patrologia Latina 91, 750-751).

Ce texte démontre clairement que, pour Bède, il est évident qu'il y a des gens dans l'Église qui reçoivent la lumière divine des mystères secrets — bien que brièvement. Ces gens sont d'une importance vitale dans l'Église comme le seraient des fenêtres dans un édifice en pierre pour qu'y jaillisse la lumière.

Le caractère contemplatif de l'ouverture à la présence de Dieu pour en être éclairé de façon personnelle et expérientielle marque le développement de la culture chrétienne du premier millénaire et au-delà. Un moine chartreux du XII ${ }^{\mathrm{e}}$ siècle, Guigues II (c. 1083-1137) a résumé le programme spirituel de sa culture de cette façon simple mais géniale:

Un jour, pendant le travail manuel, je commençai à penser à l'exercice spirituel de l'homme, et tout à coup s'offrirent à la réflexion de mon esprit quatre degrés spirituels: lecture [lectio], méditation [meditatio], prière [oratio], contemplation [contemplatio].

C'est l'échelle des moines, qui les élève de la terre au ciel. Certes, elle a peu d'échelons; elle est immense pourtant et d'une incroyable hauteur. Sa base repose sur la terre, son sommet pénètre les nuées et scrute les secrets des cieux. [...] La lecture est l'étude attentive des Écritures, faite par un esprit appliqué. La méditation est une opération de l'intelligence, procédant à l'investigation studieuse d'une vérité cachée, à l'aide de la propre raison. La prière est une religieuse application du cœur à Dieu pour éloigner des maux ou obtenir des biens. La contemplation est une certaine élévation en Dieu de l'âme attirée au-dessus d'elle-même et savourant les joies de la douceur éternelle (1970, 82-84).

Dans cette courte citation, le moine chartreux établit une distinction intéressante entre quatre étapes. La première et la deuxième requièrent une pleine activité de la personne humaine. La lectio n'est pas qu'une lecture: c'est aussi l'étude des textes. La personne spirituelle étudie les textes qui lui sont donnés par Dieu et par la tradition, afin de connaître Dieu. La meditatio est également active: la personne utilise la pleine capacité de sa propre raison humaine. Évidemment, cette méthode n'est pas une invention des Lumières: au XII ${ }^{\mathrm{e}}$ siècle, les gens étaient aussi invités à se servir de leur faculté de raisonner - sapere aude! La troisième étape met plutôt l'accent sur l'aspect relationnel: oratio veut dire "un dialogue de prière avec l'Autre». En ce qui nous concerne, la dernière étape est la plus intéressante, c'est-à-dire la contemplatio. Il n'y a dans la contemplation pratiquement pas d'activités de la personne humaine pour connaître la vérité: 
il s'agit simplement de la réceptivité pure. La vérité est donnée par l'Autre et la personne humaine la reçoit. Même si l'activité intellectuelle et l'étude méticuleuse sont de rigueur, la Vérité se révèle d'elle-même à la fin.

En examinant ce texte de près, ce qui ressort est l'équilibre parfait entre l'activité et la passivité humaine, entre la recherche et la réception. De plus, Guigues II présente une épistémologie fondamentalement relationnelle typique de la culture contemplative-mystique. L'humain et le divin sont tous deux pris au sérieux: la personne humaine recherche la Vérité absolue (c'est-à-dire: Dieu lui-même) et Dieu se révèle au moment opportun. L'oratio, le dialogue de prière, est crucial: en effet, la perspective contemplative-mystique veut que la connaissance soit relationnelle. Nos expériences quotidiennes l'illustrent bien: pour connaître l'autre, je peux l'étudier - cependant ma connaissance de cette personne serait beaucoup plus profonde, beaucoup plus intime et plus vraie si l'autre se révélait à moi personnellement. La même chose peut être dite de notre connaissance de Dieu. L'aspect épistémologique de la contemplatio implique donc une relation personnelle, un respect de l'altérité de l'autre, voire l'amour mutuel.

\subsection{Les mots «contemplatif» et «mystique»}

Avant d'aller plus loin, il convient de préciser que cette dimension contemplative fut renommée $\mathrm{au} \mathrm{Xv}^{\mathrm{e}}$ siècle: dès lors, on l'appellera «mystique». Le philosophe et historien français Michel de Certeau fut le premier à remarquer ce changement, bien qu'il le situa dans un siècle ultérieur. Les Pères de l'Église utilisaient le mot mysticus en référence aux mystères de la foi chrétienne en général; contemplatio désignait une expérience spirituelle particulière. Comme l'a remarqué Albert Deblaere, cette utilisation du mot mysticus dans un sens plus spécifique débuta au $\mathrm{Xv}^{\mathrm{e}}$ siècle à la Sorbonne dans les cours de théologie mystique de Jean Gerson, chancelier de l'Université de Paris. La tradition contemplative des premières années de la chrétienté jusqu'au Moyen Âge fut perpétuée par la tradition mystique des siècles postérieurs. La contemplation que Bède écrit est essentiellement la même que, par exemple, l'expérience mystique de Thérèse d'Avila. Au Moyen Âge, cette expérience était appelée «contemplation »; à l'époque actuelle, il est plutôt question de "mystique».

Pour résumer, on pourrait dire que la tradition contemplative ou mystique de la culture chrétienne se préoccupe de l'expérience personnelle de l'Autre divin. Bien qu'elle puisse être anticipée par des activités telles que 
l'étude ou l'utilisation autonome de la raison, l'expérience de l'Autre à travers la personne humaine est fondamentalement un don. Elle arrive lorsque Dieu se révèle comme Il est, non pas à travers la médiation des réalités créées mais immédiatement, comme dans une rencontre personnelle.

Un problème théorique est toutefois susceptible de survenir: est-ce possible de rencontrer Dieu directement? Nous avons d'innombrables témoignages de chrétiens décrivant ce type de rencontre sans intermédiaire - mais chaque contact avec Dieu n'est-il pas fait à travers la médiation? Pour répondre à ces interrogations, il convient d'examiner l'anthropologie des mystiques.

\section{L'anthropologie spécifique des auteurs mystiques des Pays-Bas au Moyen Âge}

\subsection{Trois «niveaux» ou «dimensions»}

Les auteurs mystiques des Pays-Bas au Moyen Âge avancent la même thèse qu'Augustin à ce sujet: ils distinguent trois «niveaux" ou "dimensions " de la personne humaine. La première dimension est celle du corps et des cinq sens : elle constitue le point de contact le plus évident entre la personne humaine, le monde externe et les autres créatures. Les sens perçoivent des objets significatifs parce qu'ils sont connectés aux facultés dites "supérieures ", c'est-à-dire la mémoire, la volonté et l'intellect — le deuxième «niveau » ou "dimension». Dans chaque moment et chaque perception, le passé, le présent et le futur sont implicitement donnés. Le passé est ainsi donné à travers la faculté de la mémoire; toute perception est connectée à l'immense répertoire des événements qui se sont produits dans le passé. Il en va de même pour le présent puisqu'à travers l'intellect on ne perçoit pas un chaos de couleurs, de formes, de sons et de saveurs mais bien des maisons, des personnes, des paysages, etc. Enfin, le futur nous est donné à travers la volonté: les choix sont fondamentalement reliés au futur, qu'il soit immédiat ou lointain. Les facultés supposément "supérieures» sont donc la conscience du passé, du présent et du futur dans la perception sensorielle; cette conscience est rendue possible par l'existence concrète de la personne humaine. C'est cette existence qui constitue le troisième «niveau ", le plus important en ce qui nous concerne. Les sens, la volonté, l'intellect et la mémoire présupposent l'existence; en d'autres mots, lorsque je sens ou que je goûte quelque chose, lorsque je comprends ou que je prends une décision, tout cela est rendu possible par mon exis- 
tence. C'est d'ailleurs cette thèse que René Descartes présente dans sa célèbre Deuxième méditation, la même thèse qu'avait démontrée Augustin plusieurs siècles avant lui ${ }^{3}$. L'ultime fondation de la personne humaine est l'existence du «je».

\subsection{La base de l'existence de la personne humaine en tant que relation (creatio continua)}

Toutes les suppositions susmentionnées ne sont cependant pas tellement spécifiques des mystiques chrétiens. C'est davantage dans leur examen de la profondeur du troisième «niveau» - la «dimension» fondamentale - que les mystiques vont au-delà du courant religieux majoritaire. En accord avec la théologie chrétienne qui suppose que toute chose existe parce que Dieu l'a créée, les mystiques comprennent l'existence même du «je» - le fait d'être, l'unité de l'esprit - comme une relation avec le Créateur. Dans cette perspective, la création se produit à chaque instant, non pas seulement dans le passé lointain; Maître Eckhart utilise le terme creatio continua pour désigner ce concept. Bien que chaque personne, en tant que créature, est évidemment un être réel et non pas seulement l'extension de Dieu, elle ne peut exister ne serait-ce qu'un instant sans que le Créateur n'assure son existence.

Nous avons donc déjà le premier élément de notre réponse. Est-ce possible pour une personne humaine de rencontrer Dieu directement? Chaque contact avec Dieu ne s'opère-t-il pas à travers une médiation? Selon les mystiques chrétiens, il y a toujours un contact direct entre la personne humaine et le Créateur.

Toute activité de la personne - qu'elle soit physique ou intellectuelle - présuppose une relation fondamentale dans laquelle la personne n'est pas active mais plutôt réceptive. Aucune personne ne peut s'offrir à ellemême le don de l'existence. Le fait d'être est donné, directement et immédiatement, par un Autre transcendant. Dit d'une autre façon, la fondation la plus fondamentale de la personne humaine n'est pas l'individualité du «je» mais bien une relation beaucoup plus profonde.

Cette citation de Jean Ruusbroec illustre bien ce concept:

Remarquez maintenant avec soin que chez tous les hommes se rencontre, de par la nature même, une triple unité [...] La première et la plus haute unité de l'homme est en Dieu; car toutes les créatures sont attachées à cette unité

3. Si fallor, sum (Augustin 1959, p. 114). 
divine, quant à l'essence, à la vie et à la conservation; et si, sous ce rapport, elles se séparaient de Dieu, elles tomberaient à rien et deviendraient néant. L'unité dont nous parlons est essentiellement en nous par nature, que nous soyons bons ou mauvais. Et sans notre coopération, elle ne nous rend ni saints ni bienheureux. Nous possédons cette unité en nous-mêmes, et néanmoins au-dessus de nous-mêmes, comme le principe et le soutien de notre être et de notre vie. Une seconde union existe encore en nous par nature. C'est l'unité des puissances supérieures constituée par le fait que celles-ci tirent leur origine naturelle, au point de vue de leur activité, de l'unité même de l'esprit. Il s'agit toujours d'ailleurs de cette même unité que nous possédons en Dieu; mais on la prend ici au point de vue actif, au lieu de l'envisager au point de vue essentiel. Et l'esprit est tout entier dans l'une et l'autre unité, dans la totalité de sa substance. Cette seconde unité, nous la possédons en nous-mêmes, au-dessus de la partie sensible; et d'elle procèdent la mémoire, l'intelligence, la volonté et toute possibilité d'activité spirituelle. Ici l'âme porte le nom d'esprit. La troisième unité, qui est en nous par nature, est le domaine des puissances inférieures, ayant leur siège dans le cœur comme principe et source de vie animale. [...] Ces trois unités qui sont en l'homme par nature constituent une seule vie et un seul royaume. (Opera omnia 3, 286-288; trad. par Wisques, 85)

Ruusbroec réfère aux trois niveaux; il souligne particulièrement le contact immédiat avec Dieu - contact naturel puisqu'il est manifeste dans chaque personne, qu'elle soit bonne ou mauvaise. C'est le principe même de l'être, de l'existence; nous verrons d'ailleurs que ce concept est une composante cruciale de l'anthropologie mystique. Évidemment, l'anthropologie mystique n'est pas quelque chose de nouveau: les mystiques ne font qu'exprimer une conscience particulière d'un certain élément de la théologie chrétienne. Augustin énonça ce même concept brièvement et clairement lorsqu'il affirma: Deus non fecit atque abiit, sed ex illo in illo sunt ( «Dieu ne créa pas l'univers pour s'en retirer. Ce qu'Il créa vint de Lui et demeure en Lui », Confess. 4,12,18).

\subsection{Les fondements de la personne en tant que relation transcendante («abysse»)}

L'information qui a été présentée laisse déjà entrevoir que la plus profonde réalité de la personne humaine, en tant que relation avec le Transcendant, est elle-même transcendante. Dans la tradition mystique chrétienne, on remarque souvent que Dieu, la base existentielle de la personne humaine, est comme l'abysse: sans fondement. 
Ruusbroec nous interpelle de nouveau:

On peut constater [...] que cette unité divine exerçant son attrait puissant n'est autre que l'amour sans fond, qui convie amoureusement à la jouissance éternelle le Père et le Fils et tout ce qui vit en eux. C'est en cet amour que nous voulons brûler et nous consumer sans fin, pour l'éternité, car là se trouve la béatitude de tous les esprits. C'est pourquoi nous devons établir toute notre vie sur un abîme sans fond, afin de pouvoir éternellement nous plonger dans l'amour et nous immerger dans la profondeur insondable. Et avec le même amour nous nous élèverons et surélèverons nous-mêmes jusqu'à la hauteur incompréhensible. Nous nous égarerons dans l'amour sans mode et nous nous perdrons dans la largeur sans mesure de la divine charité. Là ce sera écoulement et immersion dans les délices inconnus de la bonté et de la richesse de Dieu. Nous serons fondus et liquéfiés, engloutis et immergés éternellement dans sa gloire. Par toutes ces comparaisons je veux montrer au contemplatif ce qu'il est et ce qu'il pratique. (Opera omnia 10, 113 ; trad. par Wisques, 236)

Plusieurs observations pourraient être faites sur ce texte; j'en soulignerai deux. En premier lieu, il est clair que, pour Ruusbroec, le noyau de la personne humaine est une relation, une ouverture envers l'Autre transcendant, et que l'être humain est en soi un abysse. On retrouve d'ailleurs l'expression de ce principe dans l'architecture de presque tous les monastères contemplatifs de l'Europe médiévale. Les édifices des bénédictins et cisterciens sont tous construits sur le même modèle architectural: à la base d'un aménagement carré, on y retrouve, sur un premier côté, l'église (la dimension liturgique); sur un autre côté, la cuisine et le réfectoire; sur un troisième côté, la salle capitulaire, la bibliothèque et le scriptorium (la dimension intellectuelle); enfin, sur le dernier côté, on y retrouve des salles dédiées au travail manuel. Au centre de ce carré, il y a un espace ouvert, le hortus conclusus avec, habituellement, une fontaine au milieu. Bien que cette composition architecturale connecte les différentes dimensions de la communauté religieuse, elle symbolise d'abord la personne humaine: les quatre côtés représentent diverses activités de la personne (prier, manger et boire, travail intellectuel et manuel); l'intérieur, le centre, symbolise l'ouverture: pure, vaste, et infinie vers les altitudes, vers Dieu. Cette architecture exprime bien la pensée de Ruusbroec - la personne n'est pas fermée: dans son noyau, dans son centre, elle est grande ouverte.

Béatrice de Nazareth (1200-1268) réfère également à l'abysse qu'est la profondeur de l'âme, bien que dans son cas ce que cela signifie n'est pas immédiatement clair. Sa biographie raconte qu'elle vécut l'expérience suivante: 
Un autre jour, alors qu'elle [Béatrice] était présente au chœur parmi ses sœurs pour la célébration de la messe et qu'elle était en train de méditer les choses célestes, le cœur élevé dans une grande intensité de dévotion, voici qu'il arriva qu'un instant - le temps d'un clin d'œil — son cœur s'ouvrit par quelque fente soudaine puis se referma très rapidement pour se replacer dans sa position habituelle. La vierge bienheureuse, ne sachant pas que penser de ce mystère, se mit à demander avec insistance au Seigneur de lui montrer la cause de cette visitation momentanée ${ }^{4}$.

Cette conscience expérientielle n'est pas exclusive. Ce n'est pas que la fondation des mystiques qui est un abysse: cette réalité est vraie pour chaque personne humaine - même si sa vérité demeure cachée pour plusieurs d'entre nous.

\subsection{La fondation de la personne humaine en tant qu'abysse d'amour: l'importance de "l'altérité»}

Une autre observation concernant Ruusbroec est la suivante: il qualifie explicitement Dieu d'amour. La fondation de la personne humaine, cet abysse inscrutable, est plus spécifiquement un abysse d'amour. Au début de la citation, il dit: "cette unité divine exerçant son attrait puissant n'est autre que l'amour sans fond, qui convie amoureusement à la jouissance éternelle le Père et le Fils et tout ce qui vit en eux. " L'unité de Dieu en est une d'amour, non pas une simple identité ou uniformité. L'anthropologie de Ruusbroec est donc très conséquente avec sa théologie: le noyau de la personne humaine est une relation - une ouverture absolue au Créateur qui la crée à chaque seconde. Dieu lui-même est relation, amour absolu entre le Père et le Fils dans l'Esprit. Cette correspondance entre la théologie et l'anthropologie présente un intérêt particulier pour notre étude: pour Ruusbroec, l'union ou l'unité ne sont jamais l'uniformité. L'union de l'amour ne conduit jamais à l'uniformité parce que l'unité de Dieu (ou l'unité de la personne humaine avec Dieu), bien qu'une réelle unité, n'est

4. Alio quoque tempore, cum in choro missarum celebrati simul cum aliis interesset, et multo devotionis affectu sursum elevato corde, soli supernorum contemplationi meditando vacaret, accedit, ut repentino quodam hyatu cor illius in momento in ictu oculi, patefieret et citissime reclusum, in situ se solito relocaret. Cuius rei mysterium ignorans, virgo beatissima dominum exorare cepit attentius, ut causam illi tam momentanee visitationis ostenderet (Vita Beatricis 165, Reypens 1964, 110, trad. par Standaert, 129). 
pas une fusion, une disparition de l'un dans l'autre. L'altérité ne disparaît jamais.

Cette citation de Ruusbroec illustre bien le concept. Décrivant l'immersion en Dieu, il dit:

[...] ce n'est autre chose qu'une perpétuelle sortie de nous-mêmes avec un clair regard vers une altérité (anderheit), vers laquelle nous nous inclinons comme vers la béatitude, tout hors de nous. Nous sentons, en effet, une inclination éternelle vers quelque chose autre que nous-mêmes (Opera omnia 10, 155).

Il a été observé que Ruusbroec fut la première personne dans la culture européenne à utiliser le concept de l'altérité avec autant de précision (Bonny 2004). "Altérité » est l'antonyme d' «identité » (ou d' "uniformité »), non pas d' «unité » ou d' "union». L'antonyme d' "unité » (ou d'«union») est «séparation». Pour le mystique, l'unité présuppose l'altérité. La plus haute forme d'union n'est donc pas l'uniformité mais bien l'amour qui implique nécessairement que l'altérité ne soit pas détruite.

La réflexion des mystiques était évidemment aidée par la théologie chrétienne de la Trinité sur ce point. Le Père et le Fils sont unis dans l'Esprit — mais cela ne veut pas dire que le Père et le Fils soient identiques. Leur unité n'implique pas l'uniformité: au contraire, elle implique l'altérité. Les vieux credos proclament: clemens trinitas est una divinitas ( «la Trinité d'amour est l'unique Divinité ») ou unum deum in trinitate et trinitatem in unitate veneremur ${ }^{6}$ ( «nous honorons le Seul Dieu dans la Trinité et la Trinité dans son unité»). En d'autres termes, la compréhension chrétienne de Dieu en tant qu'amour — en tant qu'amour trinitaire — ne présente pas l'unité de la personne comme la disparition de l'un dans l'autre de sorte que l'autre cesse d'exister: l'unité est un amour qui, nécessairement, implique l'altérité de l'autre. On pourrait même affirmer que plus l'altérité de l'autre est respectée, plus l'unité d'amour est profonde.

Le lien entre l'humanité et la divinité de la personne de Jésus Christ est compris de la même façon. L'issue du débat christologique qui dura plusieurs siècles, est que l'humanité ne se fond pas dans la divinité (de façon à ce que Jésus Christ ne soit pas complètement humain) mais que chacune des deux natures restent ce qu'elles sont — que l'altérité ne disparaisse pas

5. Symbolum Clemens Trinitas ( $\mathrm{V}^{\mathrm{e}}$ ou VI ${ }^{\mathrm{e}}$ siècle, voir Denzinger et Schönmetzer 1976, n. 73).

6. Pseudo-Athanasian Symbolum Quicumque ( $\mathrm{V}^{\mathrm{e}}$ siècle, voir Denzinger et Schönmetzer 1976, n. 75). 
- bien que les natures soient pourtant unies au plus haut degré, c'est-àdire tel un unique sujet: inconfuse, immutabiliter, indivise, inseparabiliter ${ }^{7}$.

La même chose est également vraie dans la réflexion des mystiques chrétiens en matière de la maturité de l'union entre Dieu et la personne humaine. Dans cette optique, la plus haute forme d'union est l'amour: dans l'amour, l'altérité de la personne n'est pas quelque chose qui soit pris pour acquis de façon temporaire; c'est précisément la base d'une rencontre authentique. La psychologie moderne maintient qu'une relation entre deux personnes n'est ni mature ni heureuse lorsque l'un absorbe l'autre. Ruusbroec a démontré ceci très spécifiquement en affirmant que "Nous sentons, en effet, une inclination éternelle vers quelque chose autre que nous-mêmes. » Vu de cet angle, le narcissisme n'est pas la condition naturelle de la personne humaine: au contraire, "l'être » le plus profond de la personne humaine (wesen) est son «être envers l'autre» (esse ad alium). Le bonheur le plus complet de l'être humain, la béatitude suprême, est justement « une perpétuelle sortie de nous-mêmes avec un clair regard vers une altérité (anderheit), vers laquelle nous nous inclinons, tout hors de nous "; non pas rester à l'intérieur du «je», mais bien sortir de soi-même pour rencontrer l'autre en tant qu'autre.

L'anthropologie et la théologie se reflètent encore parce que, dans la perspective chrétienne, il est essentiel pour Dieu de "sortir de lui-même». Pseudo-Denys se sert d'une très belle expression lorsqu'il décrit Dieu comme une noirceur divine qui «ne peut être approchée car le débordement de ses effusions lumineuses et suressentielles la dérobent à tout regard» (Lettre 5, trad. par M. de Gandillac, 1943, 330).

\section{5 «Sortir de soi-même» comme réaction à «l'avènement de la présence de l'Autre»}

Dans la littérature mystique chrétienne, ce "sortir de soi-même» est souvent compris comme une réplique existentielle à «l'avènement de la présence de l'Autre» dans la personne humaine ( «visitation» dans le texte de Béatrice de Nazareth 1964). Un exemple clair en est donné dans la biographie d'une autre mystique du XIII ${ }^{\mathrm{e}}$ siècle, Ida de Louvain (v. 1211):

Un jour, la vierge bienheureuse ravie en extase vit sa propre âme s'étendre de l'intérieur par toutes ses parties, à l'instar d'un très large temple, et se dilater selon de fortes dimensions, tant en long qu'en large, de la façon la

7. Symbolum Chalsedonense (451), definitio (voir Denzinger et Schönmetzer 1976, n. 302). 
plus appropriée, à la ressemblance de quelque prestigieuse église, au milieu de laquelle elle prenait place; comme d'un regard perçant elle examinait tour à tour chaque détail avec une étonnante pénétration et une intense curiosité, et se réjouissait de n'avoir jamais rien vu, au monde, en toute vérité, de plus aimable et de plus beau, elle finit par tourner les yeux vers l'autel lui-même, orné, en fait, de plus noble et digne façon que tout le reste [...] Mais, de l'autre côté, elle voyait s'approcher de l'autel le pontife revêtu des insignes pontificaux [...] Il fut lui-même le vrai et suprême Pontife et Prêtre selon l'ordre de Melchisédech, le Dieu et homme parfait, né de la vierge Marie, le Seigneur Jésus-Christ. (Vie de la bienheureuse Ida de Louvain II, 34, AASS 2 avril, 180) ${ }^{8}$

Le confesseur d'Ida qui rédigea sa biographie après sa mort avait accès à de l'information de première main à propos de sa vie spirituelle et semble souvent citer presque littéralement ce qu'Ida lui a dit. Les visions qu'il mentionne reflètent une interprétation très positive de la personne humaine. L'idée que le Christ révèle sa présence dans la personne en tant que prêtre (c'est-à-dire en tant que celui qui s'offre lui-même comme don d'amour au Père) est frappante. La vie de la Trinité n'est pas quelque chose qui se produit au-delà ou au-dessus de la personne humaine: elle est présente dans le centre profond de la personne. C'est très exactement ce que Ruusbroec entend par l'expression "nous devons établir toute notre vie sur un abîme sans fond ".

Les mystiques chrétiens réfèrent souvent à la conscience de la profondeur transcendantale des fondements de l'existence comme le fait d'être «touché» (tactus). Ce qui est encore plus frappant c'est qu'ils y réfèrent également en termes de "l'arrivée de la présence de l'Autre» dans l'âme. Ida de Louvain n'est pas la seule à utiliser cette expression; il est bien connu que Maître Eckhart, un dominicain allemand (c. 1260-c. 1327), y fait explicitement référence dans son sermon Intravit Jesus in templum:

8. "Tempore quodam in excessu mentis erepta virgo beatissima vidit in spiritu suam ipsius animam ad instar templi latissimi per omnes sui partes introrsus extendi, porrectisque dimensionibus, in longum pariter et latum, ad eminentis cujusdam ecclesiae similitudinem aptissime dilatari, in cujus medio constituta, cum aciem oculorum ad singula quaeque lustranda mira sagacitate vigilique curiositate deflecteret et nullum in hoc mundo venustius se vidisse vel pulchrius aestimatione verissima jucundaret, ad ipsum tandem altare direxit intuitum, utpote ceteris omnibus honestius atque decentius adornatum. [...] Ex alio vero latere videbat indutum pontificalibus appropinquantem ad altare pontificem [...] Fuit ipse verus et summus Pontifex et Sacerdos secundum ordinem Melchisedek, perfectus Deus et homo, natus ex Maria virgine dominus Jesus Christus. » (Vita b. Idea de Lovanio 1866, II, 34, AASS aprilis 2, 180) 
Nous lisons dans le saint Évangile que Notre-Seigneur entra dans le Temple, chassa ceux qui achetaient et vendaient et dit aux autres qui offraient des tourterelles et autres choses semblables: «Enlevez cela, emportez cela!» Pourquoi Jésus chassa-t-il ceux qui achetaient et vendaient et ordonna-t-il à ceux qui avaient des tourterelles de les enlever? Il ne signifiait rien d'autre que sa volonté de voir vide le Temple, exactement comme s'il disait: J'ai un droit sur ce Temple, je veux y être seul et y avoir la souveraineté. Qu'entend-il par là? Ce Temple où Dieu veut régner en maître selon sa volonté, c'est l'âme humaine qu'il a formée et créée exactement semblable à lui-même, car nous lisons que le Seigneur dit: «Faisons l'homme à notre image et à notre ressemblance. "Et c'est bien ce qu'il a fait. Il a créé l'âme de l'homme si semblable à lui-même que ni au ciel ni sur la terre, parmi toutes les créatures magnifiques que Dieu a si merveilleusement créées, il n'en est aucune qui lui soit aussi semblable que l'âme humaine seule. C'est pourquoi Dieu veut que ce temple soit vide, afin qu'il n'y ait à l'intérieur rien d'autre que lui seul. C'est parce que ce temple lui plaît tant du fait qu'il lui est si semblable et qu'il se plaît si bien dans ce temple quand il s'y retrouve seul. (Eckhart 1958, I, 4-6)

Tout comme Ida de Louvain, Eckhart réfère à la conscience mystique de la présence du Christ en utilisant la métaphore du Christ pénétrant le temple de l'âme. En fait, l'expression «le Christ es né» dans la personne humaine est utilisée encore plus fréquemment. Dans une étude bien connue, le théologien allemand Hugo Rahner démontre que ce thème est présent dès les premiers commencements de la réflexion spirituelle chrétienne, par exemple chez Clément d'Alexandrie ou Origène (Rahner 1964). Ruusbroec a magistralement incorporé ce thème dans son livre Les Noces spirituelles (Opera omnia 3). Ce livre est écrit comme un commentaire sur un verset de l'évangile de Matthieu: "Voici l'époux! Sortez à sa rencontre!» (Mt 25,6). "Voici» réfère au caractère contemplatif et mystique de la perception spirituelle puisque la venue de l'époux est précisément ce qui peut être vue: le Christ au cœur de la personne humaine. La "sortie pour aller à sa rencontre » devient alors la réponse de la personne humaine et la rencontre en devient son accomplissement, c'est-à-dire une rencontre d'amour, d'un amour complet et mature entre celle-ci en tant que personne humaine et entre Dieu en tant que Dieu.

\subsection{Image et ressemblance: la filiation}

Nous devons explorer un peu plus loin encore puisque cette conception a une conséquence importante dans la pensée mystique. Je reviens à une 
citation que nous avons déjà lue, tirée de la biographie de Béatrice de Nazareth. Nous avons vu comment elle fait l'expérience soudaine de ses propres fondements comme d'un abysse béant, qu'elle en est surprise et qu'elle demande à Dieu de l'aider à comprendre le phénomène:

Le Seigneur très bon lui répondit aussitôt par ces paroles $[\ldots]$ : «Formons un pacte, dit-il, concluons un pacte stipulant que nous ne soyons plus séparés, mais véritablement unis» (Fedus ineamus,/Pactum pangamus/Ut decetero non dividamur / Sed veraciter uniamur). Toute réjouie à ces mots, elle fit tout de suite une réponse par laquelle elle s'obligea sur-le-champ, par engagement spontané, à observer un pacte si salutaire: "Mon cœur est prêt, dit-elle, à exécuter tout ce que tu commanderas, surtout à m'obliger moimême à observer un si grand pacte, dans lequel tu me promets, en le stipulant avec une telle liberté, de me garder en retour ton entière fidélité ». Elle avait à peine formulé cette réponse qu'il lui sembla tout de suite que le Seigneur lui-même de toute consolation et de toute miséricorde s'imprimait à lui-même toute son âme dans la plus suave étreinte, et que, tout comme une matière de cire molle marquée du sceau en garde le caractère, de même l'Esprit divin la modelait à son image à lui et lui donnait de la façon la plus gracieuse une figure à sa ressemblance9. (Vita Beatricis, 165-166; Reypens 1964, 110; trad. par Standaert, 129-130)

Afin de comprendre cette citation correctement, nous devrions discerner les différentes étapes du texte. Comme nous l'avons vu plus tôt, la première étape fut pour Béatrice de faire l'expérience de l'abysse qui est la profondeur de son être, comme si son cœur s'ouvrait soudainement (par

9. Cui per hec eadem verba, non vulgari sed latina prolata sermone sermone, pius dominus mox respondit: 'Fedus, inquit, ineamus, pactum pangamus, ut decetero non dividamur, sed veraciter uniamur.' Ad quam vocem nimis exhilarata, tale continuo responsum domino reddidit, per quod ad observationem tam saluberrimi federis, pactione voluntaria se protinus obligavit. 'Paratum, inquit, domine, cor meum ad exequendum omnia quecumque mandaveris, presertim ad obligandum memet ad observationem tanti federis, per quod mihi, fidem integram servaturum te, stipulatione tam liberrima repromittis. Qua vix responsione peracta, visum est ei continuo, quod ipse totius consolationis et misericordie dominus, illius animam, amplexu suavissimo, sibimetipsi totam imprimeret, et sicut impressa sigillo mollis cere materies, illius in se karacterem representat, sic ad ymaginem suam illam effigiatam, divinus spiritus redderet et ad similitudinem suam decentissime figuram. Vita Beatricis 165-166; Reypens 1964, 110; trad. par Standaert, 129-130; l'auteur anonyme de la Vita mentionne que la réponse du Seigneur est donnée en latin et non en langue vernaculaire. Le caractère poétique de cette citation est de fait plus évident en latin. Hadewijch renvoie au Christ comme «poète de l'amour» (Stanzaic poem 12, éd. Van Mierlo, 74, lignes 12-13). 
"cœur ", on entend le centre de la personne - non pas seulement le siège des émotions). Par la suite, c'est comme si elle entendait Dieu l'inviter à participer à une relation d'amour mutuelle. Ravie, elle donne son accord; en d'autres termes, elle ne se détourne pas de l'invitation de l'Autre divin dont elle fut surprise de découvrir la présence en elle-même. C'est ainsi qu'une relation mutuelle est établie; le texte dit alors: «l'Esprit de Dieu modela son âme selon l'image et la conforma très gracieusement à sa ressemblance avec quelque harmonie proportionnée». La phrase est courte mais elle fait référence à une intuition mystique profonde, c'est-à-dire que la personne humaine prend part à la vie de la Trinité divine.

«L'image» et la «ressemblance» sont donc des concepts clés. Dans l'anthropologie mystique, la personne humaine est créée "à l'image de Dieu » (ad imaginem). "L'image de Dieu », c'est le Christ (selon Col 1,15) et la personne humaine est créée en fonction de cette image. La personne humaine n'est cependant pas elle-même l'image de Dieu: l'image, c'est le Christ, le seul Fils du Père - et la personne humaine est intrinsèquement orientée vers le Fils et conçue pour ressembler au Fils. La ressemblance est justement la relation avec le Père, qui est une relation mutuelle d'amour. La personne humaine est ainsi conçue pour aimer et être aimée comme Jésus-Christ aime le Père et est aimé par le Père. C'est exactement ce dont Béatrice fit l'expérience: l'Esprit, qui est l'amour mutuel du Père et du Fils, lui fait ressembler à l'image qu'est le Christ. La ressemblance est basée sur l'amour mutuel.

C'est probablement là l'intuition expérientielle la plus importante de la tradition mystique chrétienne: la personne humaine participe à un amour absolu, un amour au-delà du temps et de l'espace, éternel et divin — l'union d'amour du Père et du Fils dans l'Esprit.

Plusieurs mystiques réfèrent à cette expérience. Marie d'Oignies, par exemple, est considérée comme la première représentante du mouvement mystique féminin des Pays-Bas. Son biographe, Jacques de Vitry (1160/701240) mentionne:

Un jour, alors qu'elle était dans sa cellule, elle entendit la très douce voix du Seigneur dire: Celle-ci est ma fille bien-aimée en qui je me délecte au plus haut point. (Acta Sanctorum, juin 5, 567; voir Mt 3,17: "Celui-ci est mon Fils bien-aimé en qui j’ai mis tout mon amour.» $)^{10}$

10. «Aliquando autem, dum esset in cellula sua, audivit vocem dulcissimam Domini dicentis: Haec est filia mea, in qua plurima delector ", Acta Sanctorum, juin 5, 567; voir Mt. 3, 17 Hic est Filius meus dilectus, in quo mibi complacui. 
La même sensibilité est décrite en détail par Ruusbroec:

Et c'est pourquoi, si nous sommes montés avec Jésus sur la montagne où il n'y a plus d'images en nous, si nous l'avons ensuite suivi avec un regard simple, avec une complaisance pleine de ferveur, avec une inclination fruitive, nous sentons alors la forte ardeur du Saint Esprit qui nous consume et nous fait fondre jusqu'en l'unité de Dieu. En effet, là où, ne faisant qu'un avec le Fils de Dieu, nous sommes reconduits amoureusement en notre commencement, nous entendons la voix du Père qui nous touche en nous attirant à lui, car il dit à tous ses élus en son Verbe éternel: "Celui-ci est mon Fils Bien-Aimé en qui je trouve mon bon plaisir.» En effet, vous devez savoir que du Père au Fils et du Fils au Père, leur bon plaisir s'exerce éternellement, du fait que le Fils a pris notre humanité, qu'il est mort et qu'il a reconduit tous les élus en leur commencement. Et c'est pourquoi, si nous sommes élevés par le Fils en notre origine, nous entendons alors la voix du Père nous attirant en lui, qui nous éclaire de l'éternelle vérité, et cette vérité nous montre le bon plaisir de Dieu largement ouverte, là où tout ce qui plaît commence et finit. Là, toutes nos puissances défaillent [...] et tous nous devenons un, et un seul tout, dans l'embrassement d'amour de l'Unité des Trois. (Opera omnia 10, 176; trad. par Huot de Longchamp, 41-42, modifiée)

Ruusbroec exprime la conscience d'être aimé du Père comme le Christ, le seul Fils, est aimé. Cette conscience qu'il décrit n'est pas une nouveauté: saint Paul avait déjà mentionné l'idée de la filiation dans son épître aux Romains, par exemple lorsqu'il affirma que l'Esprit — c'est-à-dire l'amour entre le Père et le Fils — nous fait clamer «Abba! Père!» $(\mathrm{Rm} 8,15)$.

Pourtant, il semble toujours étonnant que ces auteurs écrivent que la personne humaine participe complètement à l'amour trinitaire. Il serait inexact de penser que le Père nous aime moins complètement que son Fils: le Père aime son Fils et la personne humaine entièrement; la seule différence est que le Père aime le Christ naturellement (puisqu'Il est son Fils) alors qu'll nous aime par grâce. Le deuxième amour est en aucun cas moindre que le premier.

\subsection{Intermède: «l'union sans différence»}

Je me permets de faire une digression pour clarifier le concept d'union mystique en tant que supposée « union sans différence » ${ }^{11}$. C'est bien connu qu'on commença au XIII ${ }^{\mathrm{e}}$ siècle à formuler une nouvelle compréhension de l'union mystique. Bernard McGinn l'explique comme suit:

11. Pour une analyse plus poussée, voir Faesen (2011, à paraître). 
L'hérésie mystique, inconnue à l'Occident avant la fin du treizième siècle, n'était pas une intrusion étrangère, mais a surgi d'aspects inclus dans la nouvelle mystique à la fin du Moyen Âge. Un élan central de cette nouvelle vague de la mystique était le désir de parvenir à une union avec Dieu qui, à certains niveaux du moins, allait au-delà de l'union amoureuse des esprits (unitas spiritus), union qui avait tant caractérisé la mystique d'autrefois, pour atteindre un niveau de dédifférenciation, ou d'un manque de différence, entre Dieu et l'être humain. (McGinn 2006, 24)

Le premier mystique à parler d'union "sans différence » est, à ma connaissance, Hadewijch d'Anvers. La même expression se retrouve également chez Maître Eckhart qui affirme par exemple:

Nous sommes totalement transformés et changés en Dieu. De la même manière que, dans le Sacrement, le pain est transformé au corps de NotreSeigneur [...] de même je suis transformé en lui, de sorte qu'il m'opère en tant que son être, un, non pas semblable. Par le Dieu vivant c'est vrai qu'il n'y a aucune différence. (Sermon 6: Iusti vivent in aeternum, DW I, 110111 ; trad. par Ancelet-Hustache)

Nous savons que les théologiens de la cour du pape Jean XXII s'objectèrent aux travaux d'Eckhart. Nous pouvons d'ailleurs les comprendre: la rhétorique de l'union semble insinuer que les mystiques réfèrent à une véritable fusion - «Die Differenz is zurückgelassen im Vorläufigen, das

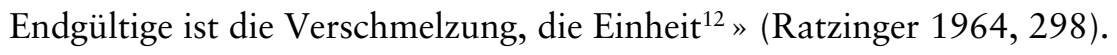

Les académiciens modernes ont cependant souvent négligé deux éléments importants à cet égard. En premier lieu, les termes «sans différence» (sine distinctione ou sine differentia) étaient une expression normale et même courante dans la théologie de la Trinité: saint Augustin l'utilise dans son De Trinitate $(97,1.20$ ), le pape Léon le Grand dans ses Traités (Tractatus 77; Chavasse 491); le plus important auteur bénédictin du XII siècle, Rupert de Deutz (Liber de divinis officiis 10; Haacke, 349, 1. 802), s'en sert également — de même que le théologien Pierre Lombard qui fut respecté universellement à travers le Moyen Âge (Sent. 2, d. 8, c. 2, par. 1; Grottaferrata, vol. I, p. 367, 1. 16). Tous ces auteurs affirment que, dans l'amour du Saint Esprit, le Père est complètement dans le Fils et le Fils est complètement dans le Père de façon à être unis sans différence ${ }^{13}$. Lorsque saint Augustin,

12. «La différence est laissée derrière à l'éphémère, l'ultime est la fusion, l'union. » (trad. par Dumas)

13. Voir la sixième lecture des Matines de la Fête de la Trinité explicite (Fulgentius, De Fide ad Petrum liber 1, 4; PL 65, 673-674): Per hanc unitatem naturalem totus Pater 
Léon le Grand et Pierre Lombard parlent d'union «sans différence », ils ne veulent fort certainement pas signifier que le Fils et le Père sont identiques; il en va de même pour le traitement de l'union divine des mystiques.

En second lieu, force est de constater que, logiquement, l'expression "sans différence» ou alors "pas de différence» réfère nécessairement à deux entités. On ne peut affirmer par exemple: «il n’y a pas de différence entre cette table", sans que la phrase soit incomplète. Il faut ajouter une comparaison: "il n'y a pas de différence entre cette table et cette autre table». Saint Augustin, Léon le Grand, Rupert de Deutz et Pierre Lombard le savaient très bien, tout comme les autres mystiques.

Cette explication est particulièrement bien articulée chez Ruusbroec. Il établit une distinction entre "altérité » et "différence », par exemple dans l'extrait suivant :

Continuellement l'esprit brûle en lui-même, car son amour est éternel, et continuellement il se sent consumer en amour, car il est attiré en la transformation qu'opère l'unité de Dieu. Là où il brûle en amour, s'il fait attention à lui-même, l'esprit perçoit distinction (onderscheet) et altérité (anderheit) entre lui et Dieu, mais là où il se consume, il est simple et ne s'en distingue aucunement, et c'est pourquoi il ne sent rien d'autre que l'unité. (Opera omnia 10, 110-112; trad. par Huot de Longchamp, 15)

Le terme «distinction» (onderscheet) désigne la différence entre soi et Dieu. "Altérité » (anderheit) réfère simplement au fait que Dieu est Autre. L'antonyme de "distinction" est donc "égalité »; celui d' "altérité » serait "uniformité » ${ }^{14}$. Ruusbroec suggère donc que lorsque la personne brûle d'amour pour Dieu, elle ressent néanmoins «distinction et altérité » cependant, lorsque la personne est consumée, la «distinction» n'est plus ressentie. Le lecteur comprend spontanément que "l'altérité », bien qu'implicite, n'est pas disparue: il n'y a toujours pas d'uniformité entre Dieu et la personne humaine. Le cas échéant, il ne pourrait plus y avoir de relation - et donc pas d'amour.

Nous devons donc comprendre correctement cette « unité sans différence». Il s'agit d'une expression que l'on situe dans le contexte de la tradition de la théologie trinitaire appliquée à la relation entre Dieu et la personne humaine. Elle ne fait pas référence à l'uniformité dans laquelle il ne peut y avoir de relation d'amour et — ipso facto — d'unité.

in Filio et Spiritu sancto est, et totus Filius in Patre et Spiritu sancto est, totusque Spiritus sanctus in Patre et Filio est.

14. N.d.T.: «sameness». 
Revenons alors sur ce qui a déjà été exposé et demandons-nous ce que tout cela signifie pour la compréhension mystique de la personne humaine.

\section{Conséquence de l'anthropologie mystique: humanisme chrétien}

\section{1 «Individu» ou «personne»}

Une des conséquences de tout ceci est cruciale pour notre sujet d'étude, c'est-à-dire l'idée que, pour le mystique chrétien, la personne humaine est une personne et non pas un individu. Le mot "personne» est relationnel alors qu' "individu " ne l'est pas. Il convient de remarquer que le concept de la "personne» fut élaboré par la pensée chrétienne à travers la conception de la relation entre le Père et le Fils. Autour de l'an 212, le théologien Tertullien fut le premier à utiliser un mot relationnel à cet égard, un mot qui était rarement utilisé dans le latin classique, c'est-à-dire persona. Le Père est Père parce qu'Il est Père d'un Fils: cette relation définit son identité; dans le même ordre d'idées, l'identité du fils consiste en sa relation avec son Père. La théologie chrétienne n'affirmerait jamais que, dans la Trinité, il y a trois «individus ». Nous référons plutôt au Père, au Fils et au Saint Esprit en tant que "personnes".

Dans la perspective des mystiques chrétiens, il vaudrait mieux utiliser le terme de "personne» qui est relationnel, plutôt que le mot «individu» en faisant référence à des êtres humains. Le mot «individu» souligne la particularité de la femme ou de l'homme qui existe en tant qu'être singulier, séparé et distinct des autres. Le mot "personne » souligne qu'elle ou qu'il est un être en relation avec d'autres; il implique également une connotation de valeur unique.

D'un point de vue historique, il convient de remarquer que deux tendances, deux interprétations de la réalité humaine se développent au Moyen Âge. Premièrement, il y a la découverte de la personne humaine en tant que "personne "; deuxièmement, il y a l'interprétation de la personne en tant qu' «individu». On ne doit pas confondre les deux puisqu'elles ont chacune leurs conséquences propres. Les mystiques soutiennent la première tendance et non pas la seconde.

La première tendance est, en fait, inhérente à la foi chrétienne. Les premiers disciples furent appelés par Jésus et vinrent à la foi de façon personnelle. "Foi» en grec $(\pi \imath \sigma \tau \imath \varsigma)$ est un terme relationnel qui pourrait également être traduit par "confiance " ou "confiance en quelqu'un d'autre ». Malgré le fait que durant la christianisation de l'Europe des tribus entières adoptaient parfois la foi chrétienne collectivement, c'est aussi 
un fait historique que cette dimension personnelle et relationnelle n'était jamais négligée. Une illustration intéressante de cette préoccupation nous provient d'une lettre envoyée à l'empereur Charlemagne en 796 par son conseiller personnel, le théologien Alcuin († 804). L'empereur venait de conquérir quelques tribus de Saxons et de Huns et les avait forcés à se convertir. Alcuin félicite poliment l'empereur et lui donne immédiatement ce conseil:

Désormais, que votre dévouement dont la haute sagesse plait à Dieu procure au peuple nouveau de pieux prédicateurs, aux mœurs irréprochables, formés à la science de la sainte foi et imbus de préceptes évangéliques; qu'ils soient aussi attentifs dans leur prédication de la parole de Dieu, aux exemples des saints apôtres qui avaient l'habitude lors de l'initiation à la foi de donner à leurs auditeurs du lait, c'est-à-dire de doux préceptes; écoutons l'apôtre Paul: «Et moi, frères, je n'ai pas pu vous parler comme à des personnes spirituelles, mais comme à des gens charnels. Comme à des tout-petits dans le Christ je vous ai donné du lait à boire, non de la nourriture solide. Vous ne pouviez pas encore en prendre, vous ne le pouvez même pas maintenant » (1 Co 3,1.2). Ce que laisse entendre ici cet homme qui a prêché au monde entier, en qui le Christ parlait, c'est qu'il faut alimenter la foi des nouveaux convertis de plus souples (préceptes) comme on nourrit de lait un bébé, de crainte qu'un esprit de santé fragile ne vomisse ce qu'il boit... Il faut aussi voir avec le plus grand soin à ce que tout se fasse dans l'ordre quant au ministère de la prédication et à l'administration du sacrement de baptême, de crainte qu'il s'avère inutile de laver le corps si dans l'âme douée de raison n'a pas précédé la connaissance de la foi catholique... Et c'est l'homme fortifié et préparé par cette foi qu'il faut baptiser. Et c'est ainsi qu'en temps opportun, à maintes reprises, les préceptes évangéliques doivent lui être dispensés par une prédication assidue, jusqu'à ce qu'il parvienne à l'âge adulte et devienne une digne demeure de l'Esprit Saint et soit un parfait fils de Dieu, comme le Père céleste est parfait, lui qui vit et règne dans la parfaite Trinité et dans l'unité bénie, Dieu et Seigneur pour les siècles des siècles. Amen. (Lettre 33, Patrologia Latina 100, c. 188-190, nous traduisons)

Pour Alcuin, il est évidemment insuffisant qu'une tribu adopte la foi chrétienne collectivement et qu'elle suive par la suite les rites et le calendrier chrétiens. Le conseiller de l'empereur est convaincu que chaque chrétien a la vocation personnelle de devenir "une demeure pour l'Esprit Saint» et «un parfait fils de Dieu». Dans la relation personnelle avec le Père, une femme ou un homme devient une personne unique et irremplaçable parce que sa relation est elle-même unique et irremplaçable. Ceci évoqua un profond et heureux sentiment de surprise dans les tribus où la 
collectivité primait sur la personne avant qu'elles adoptent la foi chrétienne au début du Moyen Âge. La découverte de la valeur unique de la personne humaine fut certainement stimulante et libératrice. Cette intuition était développée tout particulièrement par la pensée mystique et contemplative; il s'agit d'un des piliers de la civilisation de l'Europe chrétienne du début au milieu du Moyen Âge.

Il existe cependant une autre tendance dans le développement de la pensée européenne - et c'est pratiquement un mouvement contraire. Selon cette seconde perspective, l'humain est un individu: Boèce (†524/526) donne un exemple clair de ce point de vue dans sa définition de l'homme: naturae rationalis individua substantia ${ }^{15}$. Un être humain est une "substance individuelle de nature rationnelle» — pour Boèce, l'individualité fait partie de la définition de l'humain. Cette perspective est souvent rejetée par les mystiques bien qu'elle fut adoptée par des académiciens influents, dont le flamboyant Pierre Abélard (†1142/1144). Ce dernier ira même jusqu'à modifier l'idée de la "personne" dans ce sens en suggérant que l'on interprète persona en tant que per-se-una ${ }^{16}$. Pour Abélard, c'est l'individualité et la singularité qui constituent la personne humaine; nous savons que des théologiens mystiques, dont Guillaume de Saint-Thierry, s'objectaient fortement à cette perspective.

Cependant, avec la montée du nominalisme, c'est cette dernière perspective qui devient dominante. Le nominalisme soutient que les concepts abstraits — tels que «l'humain» — n'ont pas d'existence en soi, qu'ils ne sont que des mots (nomines). Nous ne rencontrons jamais «l'humain" comme tel, mais bien tel ou tel humain. Derrière cette réalisation, on découvre un important axiome: dans la perspective du nominalisme, seuls les individus existent. Chaque être coïncide complètement avec lui-même; tout ce qui existe, existe en tant qu'individu. La personne humaine n'est pas une personne mais un individu, un $« \mathrm{je}{ }^{17}$.

La différence entre ces deux interprétations est très significative. La perspective contemplative et mystique trace le chemin de l'humanisme chrétien: chaque être humain a une valeur unique parce que chacun est un lieu saint pour la rencontre de Dieu. L'autre perspective conduit à une anthropologie fermée et est individualiste. La découverte de la personne

15. «Liber de persona et duabus naturis » III, Patrologia Latina 64, c. 1343C.

16. «Expositio fidei in symbolum Athanasii », Patrologia Latina 178, cc. 631A en 624C

17. Voir Guillaume d'Ockham: quaelibet res singularis seipsa est singularis (Sent., d. II, q. vi, p, q). 
humaine en tant que personne cause un mouvement de libération au début du Moyen Âge en Europe. Cette intuition a également une force créatrice surprenante: elle donne forme à une nouvelle culture humaniste. À l'inverse, l'interprétation de la personne humaine en tant qu' "individu» ouvre la voie à des systèmes politiques et économiques dans lesquels chaque humain n'est qu'une fibre remplaçable du tissu social. Nous ne devrions jamais confondre ces deux perspectives: la découverte de l'être humain en tant que personne n'est pas un individualisme!

\subsection{Conséquences: humanisme chrétien à l'ère de la mondialisation}

Habituellement, lorsque les historiens utilisent l'expression «humanisme chrétien primitif », ils font référence au mouvement des Frères de la vie commune - à leurs écoles et à leurs éditions des classiques de la période entre 1480 et 1500 . C’est à cette période que le jeune Érasme étudia dans leurs écoles; il devint le "prince des humanistes» dont le projet de vie fut le développement d'une philosophia Christi basée sur l'étude scientifique de la Bible et des Pères de l'Église. Historiquement parlant, cet humanisme primitif puise ses racines dans la spiritualité mystique de Ruusbroec - et ce n'est pas une coïncidence: l'anthropologie mystique implique une appréciation très développée de la personne humaine.

Le point de départ de cette conviction mystique est que dans la personne humaine une rencontre d'amour se produit, une rencontre unique et transcendante. Cette conviction change la société même si elle échappe à la vérification objective. Comme l'a affirmé Émile Durkheim, la société a tendance à se répéter, à perpétuer son existence dans un égalitarisme qui considère les individus comme interchangeables. La rencontre d'amour, unique et transcendante, s'oppose à cette tendance: l'humain y découvre qu'il n'est pas un individu mais une personne (et non pas un «je» autosuffisant). La meilleure façon de contribuer à l'humanisation de la société est de prendre cette rencontre au sérieux.

La personne humaine découvre qu'elle est invitée et appelée à l'intimité avec Dieu, ce qui ne veut pas dire exécuter un programme religieux, continuer une tradition ou même poursuivre un idéal religieux. Albert Deblaere analyse certains passages clés de la règle de saint Benoît et de celle de saint Basile: il démontre que, pour ces auteurs spirituels, le but n'était pas la paix intérieure, la liberté, un plus grand contrôle des passions humaines ou encore la contemplation, mais bien une vie de communion avec JésusChrist. Cette vie de communion intime est bien entendu cachée et ne per- 
met pas de vérification objective. Il n'en demeure pas moins qu'elle est à la base du profond respect de la nature humaine qui caractérise cette longue tradition spirituelle.

Deblaere s'exprime très bien lorsqu'il affirme que chaque personne est aux yeux de l'humanisme de la tradition mystique-contemplative "une histoire d'amour qui ne sera jamais répétée»(Deblaere 2004, 194). Il s'agit, à mon point de vue, d'un excellent résumé de tout ce que nous avons étudié jusqu'à présent; il indique clairement en quoi la tradition mystique constitue un défi pour notre société mondialisée.

\section{Conclusion}

L'anthropologie de la tradition mystique-contemplative des auteurs des Pays-Bas au Moyen Âge présente effectivement la personne comme «une histoire d'amour». L'amour n'est pas quelque chose d'accidentel pour la personne humaine - bien au contraire, il s'agit de son être. L'être en soi, l'existence, est enraciné dans l'amour, l'amour transcendant de la Trinité: la personne humaine est amour.

La conscience expérientielle de cette «fondation» de la personne humaine est décrite par la tradition mystique-contemplative. Le programme de la tradition mystique chrétienne n'est pas cependant de rechercher ce genre de conscience mais plutôt d'aimer - aussi profondément et simplement que possible.

«Une histoire d'amour» implique également un développement, une chronologie, voire une aventure. C'est à cela que réfèrent les concepts d' «image» et de "ressemblance» de la tradition mystique chrétienne. L'image est donnée mais la ressemblance peut croître ou diminuer. Plus la ressemblance croît, plus la personne ressemble au Fils bien-aimé du Père, et plus sa vie devient vie - dans le sens réel du terme: une vie profonde, authentique et personnelle.

En ce sens, chaque vie humaine est unique: "une histoire d'amour qui ne sera jamais répétée ». Bien sûr, il n'existe pas deux histoires d'amour pareilles; il en va de même pour l'amour entre personnes humaines et encore plus pour l'amour entre la personne humaine et Dieu.

C'est ici que prend racine le profond respect de chaque personne humaine dans l'humanisme des mystiques. Chaque personne a une valeur unique et irremplaçable et participe d'une façon unique à l'aventure merveilleuse de l'amour transcendant. Comme l'a affirmé saint Ignace de Loyola dans ses Exercices Spirituels: "Regarder comment Dieu habite [...] 
en moi, par le don de l'être, par la vie, par la sensation et par l'intelligence. Comment il fait de moi son temple, m'ayant créé à la ressemblance et à l'image de sa divine Majesté» (Ex. Sp. 235). Cette considération s'étend à l'entièreté du genre humain.

Il s'agit d'un important défi à l'économie et à la politique de notre société mondialisée. L'anéantissement de la dignité humaine pourrait être un des drames les plus tragiques de notre ère: chaque humain pourrait être perçu comme un petit morceau remplaçable dans l'immense machine socio-économique. Nous entendons souvent dire qu'il existe dans le monde d'aujourd'hui deux types de sociétés: les individualistes et les collectivistes. Les sociétés individualistes ont tendance à mettre l'accent sur les droits individuels, la vie privée et l'autonomie, alors que les sociétés collectivistes misent sur l'harmonie générale, le devoir et le contexte social. C'est peutêtre bien vrai mais, dans les deux cas, le danger serait que l'être humain ne soit pas reconnu en tant que personne, mais seulement comme un élément remplaçable dans la machine socio-économique.

Si la société dans laquelle nous vivons était construite sur la conviction que chaque être humain est irremplaçable, non pas parce qu'il est un individu distinct, mais parce que la vocation de chaque être est de participer à l'amour transcendant, notre économie et notre politique seraient très différentes. À cet égard, les témoignages de la tradition mystique chrétienne peuvent donner l'impression d'une contre-culture.

On critique parfois la littérature mystique sous la fausse impression que les auteurs mystiques affirmeraient que la finalité de la personne humaine est de disparaître dans le divin et qu'ils n'estimeraient pas la réalité humaine (l'histoire, la culture et la société). En vérité, la tradition mystique chrétienne offre une base très intéressante pour un humanisme, c'est-à-dire une réelle appréciation de la réalité humaine. Dans la perspective des mystiques, la fondation de chaque personne humaine est l'amour absolu; la mystique a une compréhension spontanée de la valeur particulière de chaque personne. De plus, la perception relationnelle de la personne humaine en tant que "personne » invite immédiatement l'être humain à ne pas s'opposer à la communauté (comme nous pourrions le penser dans le cas d'un «individu»). En effet, celui que Ruusbroec décrit comme un mystique mature est, de fait, un bâtisseur de communauté. 


\section{Références}

\section{Sources originales et traductions}

Augustin (1959), La Cité de Dieu, Livres XI-XIV, texte B. Dombart et A. Kalb, introd. G. Bardy / trad. par G. Combès, Paris, Desclée De Brouwer (Bibliothèque Augustinienne 35).

- (1962), Les Confessions: Livres I-VII, texte M. Skutella, introd. et notes A. Solignac / trad. par E. Tréhorel et G. Boissou, Paris, Desclée De Brouwer (Bibliothèque Augustinienne 13).

- (1968), De Trinitate, texte W. J. Mountain et F. Glorie, Turnhout, Brepols (Corpus Christianorum Series Latina 50).

Beda Venerabilis, De templo Salomonis, VII, Patrologia Latina 91, 737-808.

DenZinger, H. et A. SchönmetZer (1976), dir., Enchiridion symbolorum definitionum et declarationum de rebus fidei et morum, Fribourg-enBrisgau, Herder.

Deutz, Rupert of (1967), Liber de divinis officiis, éd. par R. Haacke, Turnhout, Brepols (Corpus Christianorum Continuatio Mediaevalis 7).

Eckhart, Maître (1958), Predigten, Deutsche Werke, éd. par Josef Quint, vol. I, Stuttgart, Kohlhammer.

Guigues II le Chartreux (1970), Lettre sur la vie contemplative, intro. et texte E. Colledge et J. Walsch / trad. par un Chartreux, Paris, Cerf (Sources Chrétiennes 163).

Guillelnus De OCкнAм (1970), Scriptum in librum primus sententiarum ordinatio, éd. par S. Brown et G. Gál, Opera theologica II, New York, St Bonaventure.

Hadewijch (1942), Strofische Gedichten, texte Jozef Van Mierlo, vol. I: Tekst en commentaar; vol. II : Inleiding, Louvain, Standaard (Leuvense Studiën en Tekstuitgaven 13).

Leo Magnus (1973), Tractatus septem et nonaginta, éd. par A. Chavasse, Turnhout, Brepols (Corpus Christianorum Series Latina 138A).

Lombardus, Petrus (1971-1981), Sententiae in IV libris distinctae, Rome, Collegium S. Bonaventurae Grottaferrata (Spicilegium Bonaventarianum 4-5).

Loyola, Saint Ignace de (1960), Exercices spirituels, trad. et annot. par F. Courel, Paris, Desclée De Brouwer (Christus 5).

Migne, J.-P. (1844-1855), Patrologia Latina, Paris. 
Pseudo-Denys (1943), CEuvres complètes / trad. par M. de Gandillac, Paris, Aubier (Bibliothèque philosophique).

Ruusbroec, Jan van (1989-2006), Opera omnia, 10 vol., Turnhout, Brepols (Corpus Christianorum Continuatio Mediaevalis 101-110).

Vita Beatricis (1964), éd. par L. Reypens, Anvers, Ruusbroecgenootschap.

Vita beatae Idea de Lovanio (1866), éd. par D. Papebrochius, Acta Sanctorum aprilis 2, Paris/Rome, Palmé, c. 156-188.

Vitriaco, Jacobus de (1867), Vita beatae Mariae Oigniacensis, éd. par D. Papebrochius, Acta Sanctorum junii 5, Paris/Rome, Palmé, c. 542-572.

\section{Littérature secondaire}

Bonny, J. (2004), "Onderscheet ende anderheit bij Ruusbroec", dans Albert Deblaere, S.J. (1916-1994), Essays on Mystical Literature, éd. par R. Faesen, Leuven, Peeters (Bibliotheca Ephemeridum Theologicarum Lovaniensium 177), p. 375-388.

Bremond, H. (1938), Histoire littéraire du sentiment religieux en France, vol. IV : La conquête mystique, Paris, Bloud et Gay, 1938.

Deblaere, A. (2004), "Témoignage mystique chrétien », dans Albert Deblaere, S.J. (1916-1994), Essays on Mystical Literature, éd. par R. Faesen, Leuven, Peeters (Bibliotheca Ephemeridum Theologicarum Lovaniensium 177), p. 113-140.

FAesen, R. (2001, à paraître), "We Were Perplexed by what He Wrote": The Carthusians and a Crucial Moment in the Development of Mystical Literature in the Low Countries ", dans P. Nissen et K. Pansters, Carthusian Worlds. Exploring the Carthusians and Their Heritage, Leuven, Peeters.

McGinn, B. (2006), "The Significance of Ruusbroec's Mystical Theology », Louvain Studies, 31, p. 19-41.

Rahner, H. (1964), "Die Gottesgeburt: Die Lehre der Kirchenväter von der Geburt Christi aus dem Herzen der Kirche und der Gläubigen », dans Symbole der Kirche: Die Ekklesiologie der Väter, Salzbourg, Otto Müller Verlag, p. 13-87.

RatZinger, J. (1964), "Der christliche Glaube und die Weltreligionen ", dans J.B. Metz et H. Vorgrimmler, Gott in Welt: Festgabe für Karl Rahner, vol. II, Fribourg-en-Brisgau, Herder, p. 287-305. 


\section{Résumé}

Cet article soutient que la tradition mystique chrétienne peut contribuer de façon importante à la promotion de la dignité de la personne humaine. Puisqu'elle témoigne d'une conscience expérientielle des fondements transcendants du «je » (c'est-à-dire la rencontre de Dieu dans l'amour), l'anthropologie de la tradition contemplative et mystique préfère concevoir le fondement de la personne humaine - l'être de la personne humaine — en tant que relation d'amour transcendant plutôt qu'en termes d'individualité $\mathrm{du}$ «je». Cette relation est unique en soi; par conséquent, l'anthropologie mystique accorde de la valeur à l'unicité de chaque personne et s'oppose à l'idée qu'un humain puisse n'être qu'une individualité, une composante remplaçable de la collectivité.

\section{Abstract}

This article argues that the Christian mystical tradition may provide an important contribution to promoting the dignity of the human person. Based on the experiential awareness of the transcendental foundation of the "I» (namely the loving encounter with God), the anthropology of the contemplative-mystical tradition considers the foundation of the human person - the "being" of the human person - to be a relationship of transcendental love, rather than as the individuality of the "I». This relationship is unique and consequently, mystical anthropology emphasizes the unicity of each person and resists the notion that the human person is a mere individuality; a disposable entity in the whole. 